\title{
Fatores relacionados ao tabagismo e ao seu abandono
}

\author{
Factors related to smoking and its interruption
}

\author{
Diogo Barbalho Cardoso', Ana Paula Cota Pinto Coelho', \\ Marcelo Rodrigues ${ }^{1}$, Andy Petroianu ${ }^{2}$
}

Cardoso DB, Coelho APCP, Rodrigues M, Petroianu A. Fatores relacionados ao tabagismo e ao seu abandono. Rev Med (São Paulo). 2010 abr.-jun.;89(2):76-82.

RESUMO: Objetivo: Verificar a prevalência do hábito de fumar em um grupo profissional e evidenciar quais fatores estão relacionados ao tabagismo e ao seu abandono. Casuística e Método: Foram avaliados, prospectivamente, 494 funcionários da Procuradoria Geral de Justiça de Minas Gerais, na cidade de Belo Horizonte, consecutivos e aleatoriamente incluídos, de um total de 1229 pessoas. Eles responderam a questionário relacionado ao tabagismo, com vista à avaliação de fumantes, não-fumantes e ex-fumantes. Foram incluídas as variáveis: idade, gênero, nível educacional, estado civil, prática de atividades esportivas, religião e uso de bebidas alcoólicas. Aos fumantes foi também aplicado o teste de Fagerstrom modificado, para estabelecer o grau de dependência ao vício. Resultados: Nesta amostra, $58 \%$ dos funcionários eram do gênero feminino e $42 \%$ do gênero masculino. A idade variou entre 16 e 69 anos, com média de 36,1 $\pm 10,5$ anos. Do total pesquisado, 8,2\% eram fumantes regulares, $3,5 \%$, fumantes ocasionais, $13,3 \%$ ex-fumantes e $74,9 \%$ não-fumantes. Entre os fumantes, $76,5 \%$ tinham pouca dependência, $13,7 \%$ dependência moderada e $9,8 \%$ dependência grave. Verificou-se que a prática de religião $(p<0,001)$, independentemente da crença $(p=$ $0,642)$, e a prática de atividades esportivas $(p=0,002)$ são fatores de proteção em relação ao tabagismo. Uso de bebidas alcoólicas $(p<0,001)$ e idade mais avançada $(p=0,002)$ correlacionaram-se com o tabagismo. Entretanto, não houve associação do tabagismo com gênero $(p=0,38)$, nível educacional $(p=0,086)$, ou estado civil $(p=0,392)$. Entre os exfumantes, os três principais fatores que os levaram a parar de fumar foram: iniciativa própria $(23,4 \%)$, receio de ter doença grave $(13,83 \%)$ e odor do cigarro $(13,3 \%)$. Conclusão: Indivíduos jovens, praticantes de atividade física ou religiosa, não consumidores de bebidas alcoólicas, são menos suscetíveis ao vício do cigarro.

DESCRITORES: Tabagismo; Abandono do uso de tabaco; Grupos etários; Esportes; Bebidas alcoólicas.

\footnotetext{
1. Graduandos do curso de Medicina da Faculdade de Medicina da Universidade Federal de Minas Gerais.

2. Professor Titular do Departamento de Cirurgia da Faculdade de Medicina da Universidade Federal de Minas Gerais UFMG, Docente Livre da Faculdade de Medicina de Ribeirão Preto - USP, Docente Livre da Escola Paulista de Medicina - UNIFESP, Doutor em Fisiologia e Farmacologia, Pesquisador IA do CNPq.

Endereço para correspondência: Prof. Dr. Andy Petroianu. Av. Afonso Pena, nº 1626, Apto 1901. CEP: 30130-005, Belo Horizonte, MG. e-mail: petroian@medicina.ufmg.br
} 


\section{INTRODUÇÃO}

O tabagismo é um vício provocado pela nicotina e por outras substâncias tóxicas presentes no cigarro ${ }^{1}$, sendo a maior causa isolada e evitável de óbito no mundo ocidental ${ }^{2}$.

Estima-se que cinco a seis milhões de pessoas morrem por ano devido ao fumo ${ }^{3}$, diminuindo sua expectativa de vida em aproximadamente dez $a{ }^{4}$. Dessa forma, é de fundamental importância que programas antitabagismo sejam implementados, para prevenir doenças decorrentes do fumo, assim como ocorre no Reino Unido, que possui em seu Serviço Nacional de Saúde um programa eficaz contra o tabagismo ${ }^{5}$. Enquanto apenas $5 \%$ das pessoas que tentam parar de fumar por conta própria conseguem após 12 meses $^{6}, 15 \%$ das que seguem esse programa obtêm êxito ${ }^{7}$. Esse mesmo estudo mostrou que a terapia em grupo é melhor que o suporte individual. O papel do profissional da saúde é essencial, tendo em vista o valor do médico nesse processo. Mesmo assim, a maior parte dos profissionais negligenciam essa função ${ }^{8,9}$.

Entre os benefícios da interrupção do tabagismo, destacam-se a redução do risco de isquemia e infarto do miocárdio e a menor progressão de doenças pulmonares ${ }^{10,11,12,13}$. De modo geral, os fumantes são portadores de maior quantidade de doenças do que a população em geral ${ }^{14}$. Esse fato talvez seja um dos motivos que levam fumantes a abandonarem seu vício. De acordo com Richards et al. ${ }^{15}, 19 \%$ de ex-fumantes alegam a deterioração da saúde como a principal causa para deixar de fumar. Portanto, quem associa a sua doença ao cigarro tem maior probabilidade de interromper 0 tabagismo ${ }^{16,17,18}$.

Dentro de uma linha de pesquisa, este estudo avaliou a prevalência de tabagismo em um grupo profissional restrito, com vista a evidenciar características de fumantes, ex-fumantes e nãofumantes e identificar os fatores que contribuem para a interrupção do tabagismo.

\section{CASUÍSTICA E MÉTODO}

Este trabalho foi realizado com funcionários da Procuradoria Geral de Justiça de Minas Gerais, na cidade de Belo Horizonte. De um total de 1229 funcionários, 494, consecutivos e aleatoriamente incluídos, responderam a questionários (formulários 1 e 2) para que fosse verificada a prevalência de tabagismo nessa comunidade, sendo feita a distribuição em 4 grupos: fumantes regulares, fumantes ocasionais, ex-fumantes e não-fumantes.

Todas as pessoas que aceitaram fazer parte desta pesquisa responderam primeiramente o Formulário 1, referente à sua identificação: gênero, idade, nível educacional, estado civil, prática de atividades esportivas, religião, uso de bebidas alcoólicas e motivos que levaram ex-fumantes a abandonarem o vício. Os fumantes responderam também o Formulário 2 (teste de Fagerstrom modificado), para graduar a dependência ao fumo.

Os dados dos Formulários 1 e 2 foram submetidos à análise estatística para verificação da prevalência de fumantes, ex-fumantes e nãofumantes, e das características epidemiológicas mais comuns de cada grupo. A existência de correlação entre o tabagismo e as variáveis estudadas foi verificada utilizando o teste de Pearson. O nível de significância adotado foi de $5 \%(p<0,05)$.

O Comitê de Ética em Pesquisa da UFMG - COEP aprovou, no dia 18 de março de 2009, o projeto de pesquisa intitulado "Fatores relacionados ao abandono do tabagismo", bem como o Termo de Consentimento Livre e Esclarecido.

Formulário 1:

Por favor, responda este questionário com atenção. Ele faz parte da pesquisa "Fatores relacionados ao abandono do tabagismo" e visa a conhecer características epidemiológicas de fumantes, ex-fumantes e não fumantes na Procuradoria Geral de Justiça de Minas Gerais.

1) Idade: .................

2) Sexo: M ( ) F( )

3) Nível Educacional:

(1) Ensino Fundamental (a) Completo (b) Incompleto

(2) Ensino Médio (a) Completo (b) Incompleto

(3) Ensino Superior (a) Completo (b) Incompleto

4) Estado Civil:

(1) Solteiro

(2) Casado ou Estado Marital

(3) Separado ou Desquitado ou Divorciado

5) Pratica Atividades Esportivas? (1) Não (2) Sim Quais?

Freqüência: Semanal: ............ Dias

Horas/Dia:

6) Pratica alguma religião? (1) Não (2) Sim Qual?

7) Faz uso de bebidas alcóolicas? (1)Não

(2)Eventualmente/ Raramente

(3) 1 a 2 vezes por semana

(4)Diariamente

8) Em qual das categorias abaixo você se encaixa:

(1) Fumante regular: faz uso de 1 cigarro ou mais por dia há pelo menos 6 meses;

(2) Fumante ocasional: fuma esporadicamente ou menos de 1 cigarro por dia há pelo menos 6 meses;

(3) Ex-fumante: abandonou o cigarro há pelo menos 6 meses;

(4) Não-fumante: nunca fumou. 

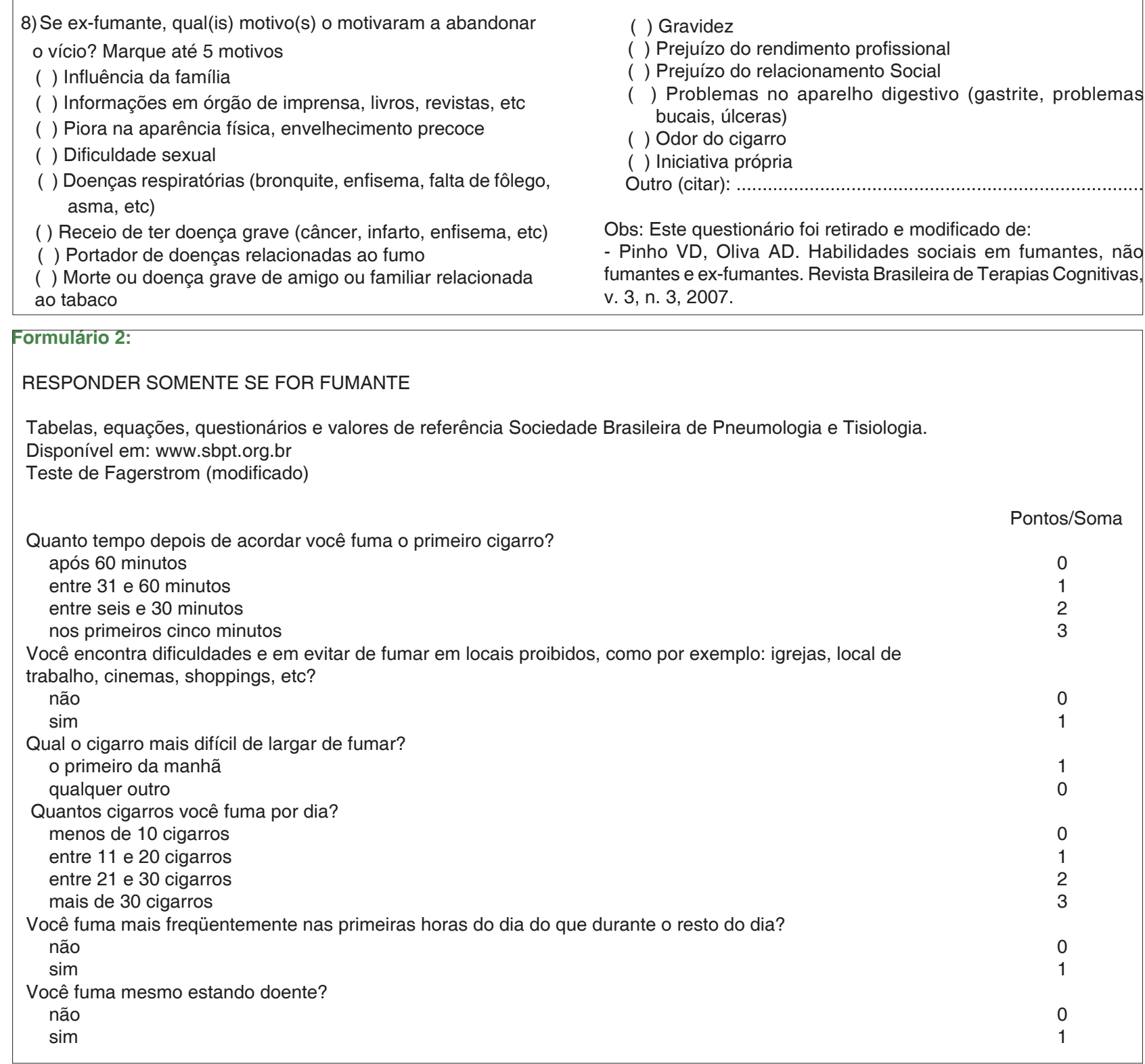

Pontuação: 0 a 4- dependência leve; 5 a 7 dependência moderada e 8 a 10 - dependência grave

Fonte: II Consenso Brasileiro de DPOC 2004 (modificado de Fagerstrom K 1989).

\section{RESULTADOS}

Os formulários foram respondidos por 494 pessoas, sendo que $8,2 \%$ eram fumantes regulares, $3,5 \%$ fumantes ocasionais, $13,3 \%$ ex-fumantes e $74,9 \%$ não-fumantes. Entre os fumantes, mediante a aplicação do teste de Fagerstrom modificado, verificou-se que $76,5 \%$ possuíam dependência leve, $13,7 \%$ dependência moderada e $9,8 \%$ dependência grave.

Entre os participantes, $58 \%$ eram do gênero feminino e $42 \%$ do gênero masculino. A idade variou entre 16 e 69 anos, com média de $36,1 \pm 10,5$ anos, com a seguinte distribuição etária: $4,6 \%$ abaixo de 18 anos, $12,2 \%$ entre 19 e 25 anos, 33,3\% entre 26 e 35 anos, $31,4 \%$ entre 36 e 45 anos, $17,0 \%$ entre 46 e 60 anos e $1,5 \%$ acima de 60 anos.

Houve associação entre idade e tabagismo $(p=0,002)$. Assim, grande parte dos fumantes regulares estava entre 46 e 60 anos de idade e a menor parte dos não-fumantes e fumantes ocasionais encontrava-se entre 26 e 35 anos. Já os ex-fumantes estavam principalmente na faixa de 46 a 60 anos.

$\mathrm{Na}$ amostra, $71,2 \%$ declararam possuir ensino superior completo, não sendo verificada correlação entre tabagismo e nível educacional $(p=0,086)$, assim como entre vício de fumar e gênero do indivíduo $(p=0,38)$, e entre tabagismo e estado civil $(p=0,392)$.

Houve correlação negativa entre o vício 
do cigarro e a prática de atividades esportivas $(p=0,002)$. Entre os fumantes regulares, $61,5 \%$ não praticavam atividade física, enquanto $63,7 \%$ dos não-fumantes eram adeptos de práticas esportivas. Destaca-se ainda que $70,8 \%$ dos que abandonaram o tabagismo praticavam exercícios físicos.

Religião também se correlacionou negativamente com o tabagismo $(p<0,001)$. Constatouse que $43,6 \%$ dos fumantes regulares praticavam atividades religiosas, enquanto $71,3 \%$ dos nãofumantes e $75 \%$ dos ex-fumantes eram atuantes em algum tipo de religião, independentemente da crença $(p=0,642)$.

Outra correlação encontrada foi com uso de álcool ( $p<0,001)$, na qual $61,3 \%$ dos não-fumantes consumiam bebidas alcoólicas, contra mais de $73 \%$ de consumo de álcool nas pessoas que possuem ou tiveram o vício do tabagismo.

Os principais responsáveis pela interrupção do tabagismo foram: iniciativa própria $(23,4 \%)$, receio de doença grave $(13,83 \%)$, odor do cigarro $(13,3 \%)$, informações em órgãos de imprensa $(7,45 \%)$, doenças respiratórias $(7,45 \%)$ e influência da família $(6,38 \%)$.

\section{DISCUSSÃO}

A prevalência de fumantes neste estudo $(11,7 \%)$ foi menor do que a divulgada em dados oficiais do Ministério da Saúde $(20,4 \%)$ de 2004, para a cidade de Belo Horizonte ${ }^{19}$. Essa diferença talvez se deva ao nível de escolaridade superior da amostra ( $71,2 \%$ dos entrevistados possuíam nível superior completo), se comparado ao da população geral. Alguns estudos reforçaram essa ideia, apresentando menor percentual de fumantes em amostras com maior nível de escolaridade ${ }^{9,20,21,22,23}$.

A religião pode ser um fator de proteção contra o tabagismo, o que também foi encontrado na literatura ${ }^{24,25}$. Segundo Dalgalarrondo et al. ${ }^{26}$, os evangélicos foram os que menos usavam tabaco, álcool e drogas ilícitas; os espíritas foram os que mais utilizavam essas substâncias; e os católicos situavam-se em posição intermediária. No presente estudo, não houve diferenças entre os tipos de crença, provavelmente devido à pequena amostragem de espíritas e evangélicos.

Com relação à idade, a frequência do tabagismo é diretamente proporcional ao avanço etário, resultado esse também encontrado por Guazelli et al. ${ }^{27}$. Esses achados reforçam o fato de que, atualmente, a juventude fuma menos, uma vez que essa é a faixa etária em que a maioria das pessoas inicia esse vício. Falcão et al. ${ }^{28}$, por exemplo, mostraram que quase metade dos tabagistas iniciaram seu vício antes dos 12 anos de idade, e mais de três quartos deles antes dos 18 anos. A redução do fumo entre os jovens talvez seja decorrente do avanço de pesquisas sobre os riscos do tabagismo, da proibição de propagandas sobre cigarro no Brasil e da implementação de programas para conscientizar as pessoas sobre os malefícios do cigarro.

O aumento do tabagismo entre mulheres fez com que não houvesse diferença na sua avaliação com relação aos homens, comportamento esse já previamente descrito por Menezes et $\mathrm{a}^{29}$ e por Rondina et al. ${ }^{30}$. Entretanto, o vício de fumar ainda é mais frequente entre os homens ${ }^{31,32,33}$.

Não houve relação entre tabagismo e estado civil, sendo o mesmo encontrado por Spada et al. ${ }^{23}$.

Cabe ressaltar que os funcionários praticantes de atividades esportivas fumavam menos, conforme já descrito por Muza et al. ${ }^{25}$. A preocupação com a saúde, característica dos que praticam esportes ${ }^{34}$, não condiz com a manutenção de vícios prejudiciais à vida. Por outro lado, o consumo de álcool associouse ao tabagismo, assim como foi descrito por Moreira et al. e por Chaieb et al. ${ }^{31,35}$. Um fato interessante verificado no presente estudo foi o de que todos os fumantes ocasionais declararam que fazem uso de bebidas alcoólicas, porém nenhum deles bebe diariamente. Isso reforça a idéia de que fumantes ocasionais fumam mais quando bebem ${ }^{36}$. Já é conhecida a tendência de quem cultiva um vício ter facilidade em adquirir outros ${ }^{37}$.

O principal motivo que levou os ex-fumantes a abandonarem esse vício foi à iniciativa própria, seguido pelo receio em ter doença grave, conforme já descrito previamente ${ }^{38}$. Esse resultado reforça a importância do papel do médico em conscientizar o paciente de que é necessário abandonar o cigarro e de que o sucesso dessa conduta depende da vontade do próprio fumante.

Um dos fatores mais relacionados com a interrupção do tabagismo foi a influência da família, o que, paradoxalmente, também contribui para o início desse vício na adolescência ${ }^{39}$. A pressão de familiares para abandonar o fumo é benéfica tanto para a saúde do fumante quanto para a saúde dos próprios familiares, visto que fumantes passivos têm maior risco de desenvolverem doenças respiratórias e eventos isquêmicos ${ }^{40,41,42,43,44}$.

Sabe-se que o odor do cigarro incomoda bastante os não-fumantes ${ }^{45}$. Neste estudo, foi possível verificar que os próprios fumantes sentiamse incomodados com o cheiro do cigarro, uma vez que esse foi o terceiro principal motivo que levou ex-fumantes a pararem de fumar.

Doenças respiratórias foram um dos motivos 
mais citados como responsáveis pelo abandono do vício, o que provavelmente resulta do conhecimento de que o fumo é a principal causa associada a doenças respiratórias graves, como bronquite crônica e enfisema pulmonar ${ }^{46,47}$.

Os meios de comunicação exercem papel positivo em relação ao abandono do tabagismo, mediante informações que esclarecem os tabagistas sobre os riscos do cigarro. Segundo os ex-fumantes, esse fato os auxiliou a interromperem seu vício.

\section{CONCLUSÃO}

Indivíduos jovens, praticantes de atividade física ou religiosa, não consumidores de bebidas alcoólicas, são menos susceptíveis ao vício do cigarro. Dessa forma, a abordagem para cessação do tabagismo deve levar em conta esses fatores.

Cardoso DB, Coelho APCP, Rodrigues M, Petroianu A. Factors related to smoking and its interruption. Rev Med (São Paulo). 2010 abr.-jun.;89(2):76-82.

\begin{abstract}
Objective: Verify the smoking prevalence in a professional group and show which factors are related to smoking and its abandonment. Methods: A prospective study evaluated 494 randomly chosen public agents from the State Justice Department of Minas Gerais, in Belo Horizonte, in a total of 1229 people. They were asked to fulfill a query related to smoking, in order to evaluate smokers, nonsmokers and former smokers. The variables included were: age, gender, educational level, marital status, sport activities, religion and alcohol consumption. Smokers were also applied to the modified Fagerstrom test in order to establish their degree of dependency. Results: $58 \%$ of participants were female and $42 \%$ male. The age ranged between 16 to 69 years old with an average of $36.1 \pm 10.5$ years old. Of the total surveyed, $8.2 \%$ were regular smokers, $3.5 \%$ occasional smokers, $13.3 \%$ former smokers and $74.5 \%$ non-smokers. Among the smokers, $76.5 \%$ presented a slight dependency, $13.7 \%$ a moderate dependency and $9.8 \%$ a severe dependency. The practice of religion $(p<0.001)$, regardless of belief $(p=0.642)$, and the practice of sports activities $(p=0,002)$ are protective factors in relation to cigarette addiction. Alcohol consumption $(p<0,001)$ and elderly age $(p=0.002)$ are correlated with smoking. However, there was no association between smoking and gender $(p=0.38)$, educational level $(p=0.086)$ or marital status $(p=0.392)$. Among former smokers, the three main factors that led them to stop smoking were their own initiative (23.4\%), fear of contracting a severe disease (13.8\%) and cigarette smelling (13.3\%). Conclusion: Youth, physical activity, religion and the non-consumption of alcohol are related with a decreased susceptibility to cigarette addiction.
\end{abstract}

KEY WORDS: Smoking; Tobacco use cessation; Age groups; Sports; Alcoholic beverages.

Agradecimentos: Agradecemos a colaboração da equipe do Serviço Médico da Procuradoria Geral de Justiça de Minas Gerais.

\section{REFERÊNCIAS}

1. West R, McNeill A, Raw M. Smoking cessation guidelines for health professionals: an update. Health Education Authority. Thorax. 2000;55(12):987-99.

2. World Health Organization. Noncommunicable Diseases and Mental Health. International Consultation on Tobacco and Youth: what in the world works? Singapore, 28-30 Sept 1999 [cited 2005 Nov 12]. Available from: https:// www.who.int/tobacco/dy_speeches7/en/

3. World Health Organization. WHO report on the global tobacco epidemic 2009: implementing smoke-free environments. Geneva: WHO Press; 2009.

4. Doll R, Peto R, Boreham J, et al. Mortality in relation to smoking: 50 years' observations on male British doctors. BMJ. 2004;328:1519.

5. Department of Health. Choosing health: making healthier choices easier. The Stationary Office; 2004.
6. Hughes JR, Shiffman S, Callas P, Zhang J. A metaanalysis of the efficacy of over-the-counter nicotine replacement. Tobacco Control. 2003;12:21-7. doi: 10.1136/ tc.12.1.21.

7. Woolacott NF, Jones L, Forbes CA, Mather LC, Sowden AJ, Song FJ, et al. The clinical effectiveness and costeffectiveness of bupropion and nicotine replacement therapy for smoking cessation: a systematic review and economic evaluation. Health Technol Assessment. 2002;6:1-245.

8. Williams GC, Levesque C, Zeldman A, Wright S, Decisão EL, et al. Health care practitioners' motivation for tobacco-dependence counseling. Health Educ Res. 2003;18:538-53.

9. Viegas CAA, Andrade APA, Silvestre RS. Características do tabagismo na categoria médica do Distrito Federal. J Bras Pneumol. 2007;33(1)76-80. 
10. Ribeiro G. Abordagem ambulatorial do paciente com DPOC e co-morbidades. Gaz Med Bahia. 2008;78 (Supl 2):52-8.

11. Chow C, Jolly S, Rao-Melacini P, Fox KAA, Anand SS, Yusuf S. Association of diet, exercise, and smoking modification with risk of early cardiovascular events after acute coronary syndromes. Circulation. 2010;121;750-8.

12. Ferrari R, Guardigli G, Tavazzi L. A modern fairy tale. Eur J Cardiov Prev R. 2009;16(Suppl 2):S65-S67.

13. Lapperre TS, Sont JK, Schadewijk A, Fox KAA, Anand SS, Yusuf S. Smoking cessation and bronchial epithelial remodeling in COPD: a cross-sectional study. Respir Res. 2007;8:85.

14. Borges M, Gouveia M, Costa J, Pinheiro LS, Paulo S, Carneiro AV. Carga da doença atribuível ao tabagismo em Portugal. Rev Port Pneumol. 2009;15(6):9511004.

15. Richards D, Toop L, Brockway K, Graham S, McSweeney B, MacLean D, et al. Improving the effectiveness of smoking cessation in primary care: lessons learned. N Z Med J. 2003;116(1173):U417.

16. Clark MA, Rakowski W, Kviz FJ, Hogan JW. Age and stage of readiness for smoking cessation. J Gerontol Soc Sci. 1997;52:S212-21.

17. Pelkonen M, Tukianen H, Tervahauta M, Notkola IL, Kivelä SL, Salorinne Y, et al. Pulmonary function, smoking cessation and 30 year mortality in middle-aged finnish men. Thorax. 2000;55:746-50.

18. Anthonisen NR. Smoking, lung function and mortality. Thorax. 2000;55:729-30.

19. Brasil. Ministério da Saúde. Instituto Nacional do Câncer. Prevalência de tabagismo no Brasil: dados dos inquéritos epidemiológicos em capitais brasileiras [citado maio 2004]. Disponível em: http://portal. saude.gov.br/portal/arquivos/pdf/tabaco_inquerito_nacional_070504.pdf

20. Vendrametto MC, Silva MC, Gomes MF. Prevalência de tabagismo em docentes de uma instituição de ensino superior. Arq Cien Saude UNIPAR. 2007;11(2):123-8.

21. Andrade APA, Bernardo ACC, Viegas CAA, Ferreira DBL, Gomes TC, Sales MR. Prevalência e características do tabagismo em jovens da Universidade de Brasília. J Bras Pneumol. 2006;32(1):23-8.

22. Zeilmann E, Nedel, F, Sandin G, Costa M. Prevalência e fatores associados ao tabagismo em uma comunidade da Região Sul de Santa Catarina, Brasil. ACM Arq Catarin Med. 2005;34(3):19-25.

23. Spada C, Treitinger A, Souza MA. Prevalência do tabagismo em doadores de sangue da região serrana de Santa Catarina - Brasil. Rev Bras Hematol Hemoter. 2006;28(1):19-23.
24. Silva LVER, Malbergier A, Stempliuk VA, Andrade AG. Fatores associados ao consumo de álcool e drogas entre estudantes universitários. Rev Saude Publica. 2006;40(2):280-8.

25. Muza GM, Costa MP. Aspectos sociofamiliares do consumo de tabaco por adolescentes escolares da rede privada do Distrito Federal. Rev ABP-APAL. 1993;15(1):31-6.

26. Dalgalarrondo P, Soldera MA, Corrêa Filho HR, Silva CA. Jovens pentecostais e espíritas em comparação a católicos: uso de álcool e drogas e saúde mental. J Bras Psiquiatr. 2005;54(3):182-90.

27. Guazzelli AC, Filho MT, Fiss E. Tabagismo entre médicos da Região do ABC Paulista. J Bras Pneumol. 2005;31(6):222.

28. Falcão TJO, Costa ICC. O tabagismo em um município de pequeno porte: um estudo etnográfico como base para geração de um programa de saúde pública. J Bras Pneumol. 2008;34(2):91-7.

29. Menezes AMB, Hallal PC, Silva F, Souza M, Paiva L, D'Ávila A, et al. Tabagismo em estudantes de Medicina: tendências temporais e fatores associados. J Bras Pneumol 2004; 30(3) 223-8

30. Rondina RC, Moratelli HB, Botelho C. Tabagismo e características da personalidade em estudantes universitários. Rev Psiquiatr Clin (São Paulo). 2001.

31. Moreira LB, Fuchs FD, Moraes RS, Bredemeir M, Cardozo S. Prevalência de tabagismo e fatores associados em área metropolitana da região Sul do Brasil. Rev Saúde Pública. 1995;29(1):46-51.

32. Nunes SOV, Onishi LO, Hashimoto SM, Kikuchi R, Toledo LGM, Koike A, et al. A história familial e a prevalência de dependência de álcool e tabaco em área metropolitana na região Sul do Brasil. Rev Psiq Clin. 1999;26(Suppl 3):84-9.

33. Paduani GF, Barbosa GA, Morais JCR, Pereira JCP, Almeida MF, Prado MM, et al. Consumo de álcool e fumo entre os estudantes da Faculdade de Medicina da Universidade Federal de Uberlândia. Rev Bras Educ Med. 2008;32(1):66-74.

34. Santos S, Knijnik J. Motivos de adesão à prática de atividade física na vida adulta intermediária I. Rev Mackenzie Educ Física Esporte. 2006;5(1):23-34.

35. Chaieb JA, Castellarin C. Associação tabagismo-alcoolismo: introdução às grandes dependências humanas. Rev Saúde Pública. 1998;32(3):246-54.

36. Rodrigues Júnior J, Ferraz S, Bruno R. Prevalência e perfil de tabagistas universitários ingressantes de uma instituição de ensino superior. Pulmão RJ. 2009;18(1):14-8.

37. De Micheli D, Formigoni ML. Are reasons for the first use of drugs and family circumstances predictors of future use patterns? Addict Behav. 2002;27(1):87-100. 
Cardoso DB, et al. Fatores relacionados ao tabagismo e ao seu abandono.

38. Santos SR, Gonçalves MS, Leitão Filho FSS, Jardim JR. Perfil dos fumantes que procuram um centro de cessação de tabagismo. J Bras Pneumol. 2008;34(9):695-701.

39. Batista AEN, Menezes LMB, Paula MLN, Falcão MA, Flores $M P$, Malta $R O$, et al. Avaliação dos fatores que contribuem para o tabagismo na adolescência. Gaz Med Bahia. 2007;77:(Supl. 1):S65-S68.

40. Brasil. Ministério da Saúde. Instituto Nacional do Câncer. Tabagismo passivo: efeitos da fumaça na saúde das crianças [citado 28 fev 2007]. Disponível em: http://www.inca.gov.br/tabagismo/frameset. asp?item=passivo\&link=crianca. $\mathrm{htm}$.

41. U.S. Department of Health and Human Services. The health consequences of involuntary exposure to tobacco smoke: a report of the surgeon general. Atlanta: U.S. Department of Health and Human Services, Centers for Disease Control and Prevention, Coordinating Center for Health Promotion, National Center for Chronic Disease Prevention and Health Promotion, Office on Smoking and Health; 2006.

42. Naeye RL. Cognitive and behavioral abnormalities in children whose mothers smoked cigarettes during pregnancy. J Dev Behav Pediatr. 1992;13(6):425-8.

43. Vineis P, Airoldi L, Veglia F, Olgiati L, Pastorelli R,
Autrup $\mathrm{H}$, et al. Environmental tobacco smoke and risk of respiratory cancer and chronic obstructive pulmonary disease in former smokers and never smokers in the EPIC prospective study. BMJ. 2005;330(7486):277.

44. Eisner MD, Balmes J, Katz PP, Trupin L, Yelin EH, Blanc PD. Lifetime environmental tobacco smoke exposure and the risk of chronic obstructive pulmonary disease. Environ Health. 2005;4(1):7.

45. Ribeiro S, Jardim J, Laranjeira R, Alves AKS, Kesselring F, Fleissig $L$, et al. Prevalência de tabagismo na Universidade Federal de São Paulo, 1996 - dados preliminares de um programa institucional. Rev Assoc Med Bras. 1999;45(1):39-44.

46. Ait-Khaled N, Enarson D, Bousquet J. Chronic respiratory diseases in developing countries: the burden and strategies for prevention and management. Bull World Health Organ. 2001;79(10):971-9.

47. Hurd SS. International efforts directed at attacking the problem of COPD. Chest. 2000;117(5 Suppl2):336S-8S.

Artigo recebido em: 02/06/2010.

Artigo aceito em: 20/06/2010. 